\section{Avaliação da oferta e utilização de especialidades odontológicas em serviços públicos de atenção secundária na Bahia, Brasil}

\author{
Assessing the supply and use of secondary \\ care in specialized dental clinics in \\ Bahia State, Brazil
}

\author{
Sônia Cristina Lima Chaves 1 \\ Denise Nogueira Cruz 2 \\ Sandra Garrido de Barros 1 \\ Andreia Leal Figueiredo 1
}

\author{
1 Faculdade de Odontologia, \\ Universidade Federal da \\ Bahia, Salvador, Brasil. \\ 2 Secretaria Estadual de \\ Saúde da Bahia, Salvador, \\ Brasil. \\ Correspondência \\ S. C. L. Chaves \\ Departamento de Odontologia \\ Social, Faculdade de \\ Odontologia, Universidade \\ Federal da Bahia. \\ Av. Araújo Pinho 62, Salvador, \\ BA 40116-900, Brasil. \\ schaves@ufba.br
}

\begin{abstract}
This study aimed to evaluate the utilization of dental services in four specialized dental clinics in Bahia State, Brazil, and to identify associated factors. This was an evaluative study in which the quantitative stage focused on a cross-sectional analysis of secondary data from the Outpatient Information System of the Unified National Health System and the qualitative component was based on 20 semi-structured interviews with coordinators and managers, in addition to on-site observation of health services. The study showed good results in the supply of these services from the health professionals' perspective, but with a low utilization rate. The low utilization rate reveals access barriers in the health services themselves, like the lack of performance standards and goals by specialty, lack of patients (who are not replaced), and different technologies used by each specialist. The results suggest the need for studies that analyze different forms of services organization that would have allowed greater utilization of the available supply, with pay differentials, linkage to primary care, and adjustment of the supply to the population's health needs.
\end{abstract}

Dental Specialities; Dental Health Services; Health Evaluation

\section{Introdução}

Os avanços tecnológicos, o uso da boa técnica e a utilização de pessoal auxiliar têm sido relacionados com uma maior capacidade dos cirurgiões-dentistas em produzir mais serviços odontológicos por unidade de tempo ${ }^{1}$, especialmente nos modelos de livre mercado como o norte-americano. No entanto, em modelos de atenção à saúde públicos ou mistos, como na Noruega ou Reino Unido tal argumento não é suficiente para explicar maior produtividade, aqui entendida como sinônimo de maior utilização de serviços 2,3.

Além disso, uma maior oferta e disponibilidade de serviços de saúde podem não necessariamente significar maior utilização 4,5 . As principais razões para a distância entre oferta e utilização podem estar associadas à disponibilidade da força de trabalho e sua distribuição geográfica, às mudanças no perfil epidemiológico que reduz necessidades, à facilidade de acesso a serviços odontológicos como redução de barreiras financeiras, geográficas e, especialmente as características da gestão do serviço ${ }^{6,7}$ como a natureza do prestador (público ou privado) e a forma de remuneração pelo serviço (se assalariada, per capita ou pós-fixada pelo rendimento) 2,8 . Esses fatores podem influenciar o desempenho do serviço no cumprimento dos padrões de oferta adequada de procedimentos. 
Sabe-se que a utilização dos serviços de saúde é resultante da interação do comportamento do indivíduo que procura os cuidados, das características do serviço de saúde e do profissional que o conduz dentro do sistema de saúde, especialmente nos serviços especializados 9,10.

No Brasil, a discussão quanto ao acesso à atenção odontológica especializada no sistema público é recente 11,12 e torna-se relevante na medida em que a atual Política Nacional de Saúde Bucal (PNSB), denominada Brasil Sorridente tem visado, entre outros objetivos, viabilizar a oferta da atenção secundária através, principalmente, dos Centros de Especialidades Odontológicas (CEOs) ${ }^{13}$. Os CEOs são um tipo de serviço de saúde que deve oferecer à população, no mínimo os serviços de diagnóstico bucal, com ênfase no diagnóstico e detecção do câncer de boca, periodontia especializada, cirurgia oral menor dos tecidos moles e duro, endodontia e atendimento a pacientes com necessidades especiais.

Em países como o Brasil, o acesso à atenção odontológica apresenta profunda desigualdade e há controvérsias se o investimento em serviço especializado é a solução adequada haja vista que a maior parte da redução dos principais agravos bucais (cárie dentária e doença periodontal) está no acesso à atenção primária (acesso ao flúor e disseminação das práticas de higiene oral) $14 \mathrm{e}$ melhoria nos indicadores sociais (escolaridade, renda e ocupação), e não necessariamente na assistência odontológica propriamente dita 15,16, como também tem sido apontado em estudos críticos na área da epidemiologia social sobre o cuidado médico 17. Ainda assim, em países com escasso acesso ao cuidado odontológico, a formulação de políticas que visam ampliar acesso à prática restauradora, inclusive especializada, é necessária já que a prática odontológica pública ainda está centrada em extrações dentárias e poucas práticas preventivas 15 .

Estudos avaliativos sobre a implementação dos CEOs discutem os seus custos como próximos àqueles da atenção primária pelo predomínio dos gastos com pessoal 12, mas também apontam uma baixa performance desses serviços. Um estudo desenvolvido em Pernambuco apontou que apenas 40,9\% dos CEOs cumpriam com um bom desempenho as metas de produção ambulatorial propostas pelo nível central, formulador e indutor da política 11 .

Como no Brasil, na Bahia, a expansão da oferta de serviços odontológicos especializados é recente. Deste modo, o presente estudo teve como objetivo avaliar a oferta e a utilização dos serviços odontológicos especializados de quatro CEOs da Bahia, considerando que possivelmente a oferta disponível está sendo subutilizada pelos usuários potenciais, identificando possíveis fatores relacionados, bem como propor um padrão de produção ambulatorial por especialidades nessa área.

\section{Metodologia}

\section{Desenho do estudo}

Esta foi uma pesquisa avaliativa com abordagem qualitativa e quantitativa. O componente quantitativo foi um estudo transversal a partir dos dados secundários de produção ambulatorial do Sistema de Informações Ambulatoriais do Sistema Único de Saúde (SIA-SUS) em que foram confrontadas a oferta disponível e a utilização. Foram estudados quatro CEOs situados em municípios da Bahia participantes de quatro estudos de caso em um estudo mais amplo de avaliação da atenção especializada na Bahia. Os critérios de inclusão dos CEOs foram: (a) portaria de habilitação ministerial publicada há pelo menos um ano e meio; (b) produção mínima de procedimentos de média complexidade no SIA-SUS no ano de 2007/2008; (c) indicação pela área técnica de saúde bucal do gestor estadual de municípios considerados exitosos na implementação dos CEOs no estado e (d) gestão municipal.

Todos os municípios selecionados apresentaram, no momento da coleta de dados, apenas um CEO. Quanto ao porte populacional, três municípios (A, B e D) eram de médio porte - de 21.000 a 100.000 habitantes - e um de grande porte - acima de 100.000 - (município C) (Instituto Brasileiro de Geografia e Estatística. http:/ /www.ibge. gov.br, acessado em 04/Abr/2008).

O componente qualitativo exploratório foi realizado através de entrevista semi-estruturada junto ao coordenador do CEO no município, dois especialistas e dois auxiliares de saúde bucal atuantes por CEO, totalizando vinte entrevistas; além de observação in loco do serviço. As principais dimensões e critérios para avaliação consideraram as seguintes categorias: (a) os principais resultados identificados pelos gestores e executores; (b) a organização do serviço, incluindo o número de atendimentos diários por especialidade, a existência de padrões propostos e o tempo médio dos procedimentos por paciente; (c) a articulação com a atenção básica (Tabela 1). Os resultados foram analisados pela técnica de análise de conteúdo ${ }^{18}$. As entrevistas foram gravadas, transcritas e analisadas no seu conjunto, identificando os principais pontos convergentes e divergentes entre os casos. 
Principais dimensões e critérios para análise da implementação da atenção secundária na Política Nacional de Saúde Bucal (PNSB). Bahia, Brasil, 2008.

\begin{tabular}{|c|c|c|}
\hline Dimensão & Critério & Fonte de evidência \\
\hline Percepção dos resultados & $\begin{array}{l}\text { Os principais resultados } \\
\text { identificados }\end{array}$ & $\begin{array}{l}\text { Entrevista: "Na sua opinião, quais os principais resultados observados por você } \\
\text { aqui no serviço de saúde?" }\end{array}$ \\
\hline Gestão e práticas & A organização do serviço & $\begin{array}{l}\text { Entrevista: "Descreva as atividades que você desenvolve. Cite exemplos", } \\
\text { "Qual o número de atendimentos diários feitos por você nesse serviço?", } \\
\text { "Há um padrão proposto pelo serviço de produção diária ou mensal?" } \\
\text { Observação in loco: tempo de consulta, horário de início e fim das atividades } \\
\text { do turno de trabalho }\end{array}$ \\
\hline & $\begin{array}{l}\text { A articulação com a atenção } \\
\text { básica e os protocolos } \\
\text { assistenciais }\end{array}$ & $\begin{array}{l}\text { Entrevista: "Como se organiza o acesso ao CEO no município } \\
\text { (a articulação com a rede básica)?" }\end{array}$ \\
\hline
\end{tabular}

\section{Conceitos utilizados}

A oferta potencial foi aqui compreendida como a disponibilidade de recursos para produção de procedimentos por parte do serviço 19.

Nesse sentido, a oferta potencial foi entendida em três sentidos. A primeira oferta potencial em saúde bucal se refere a quanto o conjunto de cirurgiões-dentistas do CEO poderiam produzir em termos de procedimentos, considerando sua carga-horária mensal, retirando-se um mês de férias. Ou seja, a capacidade potencial de produção dos profissionais de saúde bucal segundo estimativa de produção ambulatorial da Portaria $G M n^{\circ} .1 .101 / 200220$.

A segunda oferta foi aquela calculada conforme o conjunto de cirurgiões-dentistas do CEO poderia produzir em termos de procedimentos, considerando sua carga-horária mensal, retirando-se um mês de férias a partir do padrão de procedimentos propostos pela consulta aos experts.

A terceira oferta foi aquela calculada a partir dos padrões propostos para habilitação dos CEOs (Portarias GM $n^{o}$. 1.571/2004 e GM $n^{o}$. 1.572/2004) 21,22.

A utilização do serviço se refere a quanto o conjunto dos cirurgiões-dentistas do CEO por especialidade realmente produziu em número de procedimentos registrados no SIA-SUS, ou seja, que foram utilizados pelos usuários potenciais desse serviço.

\section{Coleta e análise dos dados}

A coleta de dados para a "utilização" realizou-se por meio do levantamento da produção ambulatorial dos quatro CEOs a partir dos arquivos disponibilizados do SIA-SUS e tabulados pelo programa TabWin (Departamento de Informática do SUS. http://portal.saude.gov.br/portal/se/ datasus/area.cfm?id_area=732). Foram listados todos os procedimentos odontológicos registrados, bem como a quantidade de cada um destes, segundo o estabelecimento de saúde no ano de 2008.

Em seguida, partiu-se para classificação dos procedimentos realizados em três grupos: periodontia, cirurgia e endodontia. Vale ressaltar que os demais procedimentos também foram agrupados (procedimentos básicos, de prótese, de imagem e consulta); mas, neste estudo, optou-se por analisar os grupos que compõem as especialidades mínimas para o funcionamento do CEO.

A Tabela 2 apresenta a síntese dos códigos registrados e a distribuição dos mesmos segundo os grupos adotados.

Em seguida, foi calculada a oferta ou disponibilidade do serviço por especialidade utilizando como valor de referência o padrão proposto pelo Ministério da Saúde (Portaria GM $n^{o}$. $1.101 / 2002) 20$ e o padrão proposto por consulta aos experts, criado para este estudo e explicitado a seguir, bem como o número de procedimentos por especialidade e por tipo de CEO das Portarias GM $n^{o}$. 1.571/2004 e GM $n^{o}$. 1.572/2004 21,22.

Para avaliar a relação entre a oferta e a utilização dos serviços odontológicos especializados, foi calculada para cada um dos CEOs a disponibilidade a partir da capacidade de oferta de serviço dos cirurgiões-dentistas contratados segundo sua carga horária e, finalmente, a utilização, que se refere aos procedimentos registrados como realizados no SIA-SUS.

A taxa de utilização é apresentada em forma percentual e traduz a relação entre a utilização ou uso e a oferta. Assim, quanto mais próxima 
Relação de códigos do Sistema de Informações Ambulatoriais do Sistema Único de Saúde (SIA-SUS) por especialidade registrados nos Centros de Especialidades Odontológicas (CEOs) estudados. Bahia, Brasil, 2008.

\begin{tabular}{|c|c|}
\hline Especialidade & Códigos \\
\hline \multirow[t]{5}{*}{ Cirurgia } & 0201010232; $0201010348 ; 0201010526 ; 0301100152 ; 0401010031 ; 0401010058 ; 0401010066$; \\
\hline & 0401010082; 0404020054; 0404020089; 0404020097; 0404020100; 0413040151; 0414010299; \\
\hline & 0414010310; 0414020014; 0414020049; 0414020057; 0414020103; 0414020138; 0414020146; \\
\hline & 0414020251; 0414020260; 0414020278; 0414020286; 0414020294; 0414020308; 0414020340; \\
\hline & $0414020359 ; 0414020383 ; 0414020405 ; 0414020030 ; 0414020200$ \\
\hline \multirow[t]{2}{*}{ Endodontia } & 0307010015; 0307020010; 0307020029; 0307020037; 0307020045; 0307020053; 0307020061; \\
\hline & 0307020070; 0307020088; 0307020096; 0307020100; 0307020118; 0414020022; 0414020073 \\
\hline \multirow[t]{2}{*}{ Periodontia } & 0307030016; 0307030024; 0307030032; 0414010019; 0414020081; 0414020154; 0414020162; \\
\hline & 0414020375 \\
\hline
\end{tabular}

de $100 \%$, melhor essa relação e, portanto, mais adequada para o serviço. Uma taxa de utilização em torno de $85-95 \%$ é considerada adequada. Contudo, quando essa taxa supera estes percentuais, pode estar revelando alto desempenho ou inadequação dos padrões propostos.

\section{Consulta a experts}

A opinião de especialistas na definição de critérios e padrões de qualidade é recomendada quando não existem normas sobre a temática. Na saúde bucal, o padrão de procedimentos sugere uma oferta de três procedimentos por hora na Portaria GM no. 1.101/2002 20. Assim, foi constituído um comitê de três especialistas por área (cirurgia oral menor, periodontia e endodontia) quanto à opinião dos mesmos com relação ao número possível de procedimentos especializados a serem realizados por hora, totalizando a consulta a nove profissionais. O método utilizado para o consenso foi o método Delphy 23,24, que garante o anonimato dos especialistas. Cabe destacar que todos eram especialistas atuantes na clínica e/ou professores das disciplinas relacionadas, portanto, com legitimidade para discorrer sobre a temática.

Cada um dos profissionais recebeu um questionário contendo os procedimentos do SUS segundo sua respectiva especialidade odontológica. Os experts opinavam sobre a produtividade máxima de cada um dos procedimentos a cada hora de trabalho do cirurgião-dentista no CEO. Depois disso, era calculada a média de procedimentos por hora por especialista consultado e, posteriormente, a média aritmética entre os especialistas de cada área, bem como seu respectivo desvio-padrão (DP). Ou seja, na endodontia, o especialista opinava que era possível realizar dois acessos à polpa dentária por hora, mas apenas uma obturação de dente permanente uniradicular e assim sucessivamente. Esse total de procedimentos por hora foi somado e produziu uma média aritmética de procedimentos possíveis por hora de cada especialidade.

\section{Aspectos éticos}

O projeto foi submetido e aprovado pelo Comitê de Ética em pesquisa do Instituto de Saúde Coletiva da Universidade Federal da Bahia (ISC/ UFBA) (registro CEP-ISC 005-07, aprovado em 31 de maio de 2007). Os dados secundários foram oriundos da base nacional do DATASUS (Departamento de Informática do SUS. Sistema de Informação Ambulatorial do SUS. http://www. datasus.gov.br, acessado em 02/Fev/2010) e para a aplicação dos questionários e entrevistas foi solicitado de todos os experts, gestores e profissionais dos CEOs o termo de consentimento livre e esclarecido acerca de sua participação voluntária.

\section{Resultados}

O estudo revelou que as taxas de utilização variaram conforme o padrão utilizado, com valores acima de $100 \%$ no caso do padrão proposto pelas portarias específicas de habilitação dos CEOs 21,22 e muito baixas segundo o padrão proposto pela Portaria GM $n^{o}$. 1.101/2002 do Ministério da Saúde 20 . Ou seja, utilizando-se os valores de referência das normas de implantação dos CEOs acima descritas, as taxas de utilização são muito elevadas, com valores sempre acima de $100 \%$ 
nas especialidades de endodontia e periodontia, chegando até a $512,4 \%$ na endodontia no município A. Apenas para a cirurgia oral menor, as taxas de utilização foram mais baixas utilizandose esse padrão de referência.

Conforme o padrão proposto pelo Ministério da Saúde na Portaria GM $n^{o}$. 1.101/2002 20, as taxas de utilização revelaram-se muito baixas e com variação em todos os CEOs estudados, com valores na endodontia entre $13,7 \%$ no município B e $33,4 \%$ no município C. Na cirurgia oral menor variou entre $21,2 \%$ no município A e $41,3 \%$ no município $\mathrm{B}$, com valores muito abaixo do esperado. Contudo, as taxas de utilização da periodontia sempre se mostraram mais elevadas; entre $73,1 \%$ e $105,7 \%$ (Tabela 3 ).

A consulta a especialistas quanto à revisão do padrão de produção ambulatorial por hora por especialidade permitiu observar que a média de procedimentos por hora a ser realizado segundo cada uma das especialidades analisadas e o respectivo DP foi inferior aquele proposto pelo Ministério da Saúde para a atenção ambulatorial, que é de três procedimentos por hora. Para endodontia, a média aritmética entre os especialistas foi 1,3 procedimento por hora ( $\mathrm{DP}=0,3$ ); para periodontia, 1,5 procedimento por hora $(\mathrm{DP}=0,5)$ e para cirurgia oral menor, 1,5 procedimento por hora ( $\mathrm{DP}=0,35$ ). Observou-se um baixo dissenso entre os especialistas haja vista o desvio padrão ser inferior a 0,5.

Essa diferença influenciou as taxas de utilização dos procedimentos segundo o padrão proposto pelos especialistas. Na periodontia, a taxa de utilização variou entre $97,5 \%$ no CEO do município C a 209,8\% no CEO do município A. Na endodontia, a taxa de utilização foi de 25,8\%, $31,6 \%, 47 \%$ e 77,1\% no CEO dos municípios D, B, A e C, respectivamente. Na cirurgia oral menor, a taxa de utilização foi de $32,2 \%$ no CEO do município D e de 82,5\% no município B.

Chamou a atenção que na especialidade periodontia, mais de $95 \%$ dos procedimentos deste grupo corresponderam à raspagem, alisamento e polimento sub e supragengival e raspagem corono-radicular (Tabela 4), procedimentos típicos da atenção primária, que não deveriam ser referenciados à atenção secundária ou deveriam ser referenciados com precaução. Isso provavelmente explica as altas taxas de utilização encontradas nessa especialidade.

Portanto, as taxas de utilização segundo a proposta das portarias de habilitação do CEO são bastante elevadas quando comparadas aos demais padrões de oferta sugeridos. Ou seja, todos os CEOs praticamente cumpriram os padrões propostos pelo Ministério da Saúde em 2004 nas três especialidades básicas presentes no CEO.
Entre os quatro CEOs analisados, aquele que apresentou melhor desempenho global, considerando as três especialidades foi o CEO do município C, ou seja, do município de grande porte, seguido pelo CEO do município A, exceto para cirurgia oral menor. Contudo, as variações foram muito grandes entre eles.

Como relatado anteriormente, na análise ao interior das especialidades, chamou a atenção na periodontia, predominância quase absoluta em todos os CEOs dos procedimentos de "raspagem corono-radicular" "raspagem alisamento supragengival" e "raspagem alisamento subgengival" (entre 92,1\% dos procedimentos de periodontia no município C e 99,4\% no município A). Ou seja, procedimentos típicos da atenção primária (Tabela 4).

Na endodontia, chamou a atenção a não realização de obturação em molares permanentes em três dos quatro CEOs analisados. Quanto à cirurgia oral menor, há uma distribuição mais igualitária entre os procedimentos mais comuns dessa especialidade como remoção de dente incluso, variando entre $12,5 \%$ a $23,9 \%$ do total de procedimentos dessa especialidade (Tabela 4). Contudo, a realização de excisão e sutura de lesões na boca (sugestivo de procedimento diagnóstico de câncer de boca), variou de $24,8 \%$ do total de procedimentos de cirurgia oral menor no município A e apenas $2,1 \%$ e $0,1 \%$ dos procedimentos nessa especialidade no município D e B, respectivamente. Além disso, observou-se uma grande proporção de registro de remoção de foco residual, procedimento mais recomendado para a atenção primária (Tabela 4).

Entre as prováveis explicações para os resultados encontrados, a análise qualitativa revelou que não há um padrão proposto pelo serviço de produção por procedimentos. Geralmente, há referência ao descrito nas portarias ministeriais e em alguns casos, é um padrão por número de pacientes. Não há clareza das metas a serem cumpridas pelos serviços, onde o termo procedimentos por especialidade é compreendido, no caso da endodontia, como apenas a obturação final do canal radicular, muitas vezes pelo próprio coordenador.

“...Eles normalmente seguem a normatização do Ministério, 150, 170, e não sei o quê" (Coordenador, município A).

"Varia de especialidade para outra, mas em endodontia são oito pacientes por dia, cirurgias são mais, acho que dez pela manhã e cinco à tarde..." (Coordenador, município D).

"Endodontia são quatro pacientes/turno. A Periodontia dá em torno de 6 a 8 pacientes assim, marcados. (...) a cirurgia atende assim: faz tantas cirurgias: 3 ou 4. Mas, exame ele faz o que está lá 
Oferta de procedimentos segundo padrão do Ministério da Saúde (Portarias GM n. 1101/2002 20, GM n. 1571/2004 21 e GM n. 1572/2004 22) e da consulta aos especialistas, utilização e taxa de utilização nas especialidades básicas de Endodontia, Periodontia e Cirurgia Oral Menor em quatro Centros de Especialidades Odontológicas (CEOs). Bahia, Brasil, 2008.

\begin{tabular}{|c|c|c|c|c|}
\hline Especialidade & $\begin{array}{l}\text { Município A * } \\
\text { (CEO tipo I) }\end{array}$ & $\begin{array}{l}\text { Município B * } \\
\text { (CEO tipo I) }\end{array}$ & $\begin{array}{l}\text { Município C * } \\
\text { (CEO tipo III) }\end{array}$ & $\begin{array}{l}\text { Município D * } \\
\text { (CEO tipo II) }\end{array}$ \\
\hline \multicolumn{5}{|l|}{ Endodontia } \\
\hline Segundo Portaria $n^{\circ} .1 .101 / 2002$ ** & 10.560 & 5.280 & 10.560 & 7.920 \\
\hline 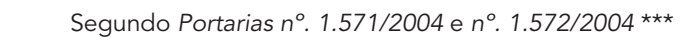 & 420 & 420 & 1.140 & 720 \\
\hline \multicolumn{5}{|l|}{ Taxa de utilização (em \%) } \\
\hline Segundo Portaria no. 1.101/2002 & 20,4 & 13,7 & 33,4 & 14,9 \\
\hline Taxa de utilização experts & 47,0 & 31,6 & 77,1 & 25,8 \\
\hline Segundo Portarias $n^{\circ} .1 .571 / 2004$ e $n^{\circ} .1 .572 / 2004$ & 512,4 & 171,9 & 309,6 & 164,0 \\
\hline \multicolumn{5}{|l|}{ Periodontia } \\
\hline Utilização no SIA-SUS & 2.770 & 2.640 & 2.573 & 1.861 \\
\hline \multicolumn{5}{|l|}{ Taxa de utilização (em \%) } \\
\hline Segundo Portaria n. 1.101/2002 & 104,9 & 50,0 & 73,1 & 105,7 \\
\hline Taxa de utilização experts & 209,8 & 100,0 & 97,5 & 70,5 \\
\hline Segundo Portarias $n^{0} .1 .571 / 2004$ e $n^{0} .1 .572 / 2004$ & 384,7 & 366,7 & 142,9 & 172,3 \\
\hline \multicolumn{5}{|l|}{ Cirurgia oral menor } \\
\hline \multicolumn{5}{|l|}{ Oferta (em número de procedimentos) } \\
\hline Segundo Portaria $n^{\circ} .1 .101 / 2002$ & 2.640 & 5.280 & 2.640 & 3.960 \\
\hline Segundo Portarias $n^{\circ} .1 .571 / 2004$ e $n^{0} .1 .572 / 2004$ & 960 & 960 & 1.320 & 2.640 \\
\hline Segundo padrão experts & 1.320 & 2.640 & 1.080 & 1.080 \\
\hline
\end{tabular}

SIA-SUS: Sistema de Informações Ambulatoriais do Sistema Único de Saúde.

* Município A, B, D: médio porte; Município C: grande porte;

** O cálculo considera a carga horária dos profissionais realizando três procedimentos por hora durante 11 meses no ano (um mês de férias);

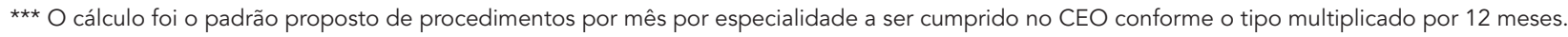
Exemplo: endodontia exigia 35 procedimentos mês multiplicado por 12 meses, totalizou 420 procedimentos como oferta potencial no ano;

\# O cálculo considera a carga horária dos profissionais realizando 1,2 a 1,5 procedimentos por hora (segundo especialidade) durante 11 meses no ano (um mês de férias), conforme consulta aos especialistas.

e vai agendando de acordo com prioridade, quem tiver maior necessidade. Aí estes exames varia muito. 8, 10, depende de quantos já foram encaminhados" (Coordenador, município E).

“...Na quantidade de pacientes não é obrigatório, na verdade, a gente tem que cumprir uma quantidade de procedimentos por mês, a gente acaba controlando a quantidade de pacientes diários" (Endodontista, município C).
Aliado a isso, no componente da organização do serviço, a falta constante de pacientes parece também contribuir para a redução da taxa de utilização desses serviços porque não há substituição de faltosos, ou marcação extra, prevendo tal ocorrência.

“Todos os endodontistas têm uma cota de seis pacientes por turno para uni-radiculares. Algumas vezes a gente se depara com faltas e ausentes, 
Procedimentos realizados (utilização) por grupo nas especialidades básicas de endodontia, periodontia e cirurgia oral menor em quatro Centros de Especialidades Odontológicas (CEOs). Bahia, Brasil, 2008.

\begin{tabular}{|c|c|c|c|c|c|c|c|c|}
\hline \multirow[t]{2}{*}{ Procedimentos por especialidade } & \multicolumn{2}{|c|}{ Município A } & \multicolumn{2}{|c|}{ Município B } & \multicolumn{2}{|c|}{ Município C } & \multicolumn{2}{|c|}{ Município D } \\
\hline & $\mathbf{n}$ & $\%$ & $\mathbf{n}$ & $\%$ & $\mathbf{n}$ & $\%$ & $\mathbf{n}$ & $\%$ \\
\hline \multicolumn{9}{|l|}{ Periodontia } \\
\hline $\begin{array}{l}\text { Raspagem e alisamento supragengival, subgengival } \\
\text { e corono-radicular (por sextante) }\end{array}$ & 2.754 & 99,4 & 2.596 & 98,3 & 2.369 & 92,1 & 1.828 & 98,2 \\
\hline Gengivectomia e gengivoplastia (por sextante) & 9 & 0,3 & 36 & 1,4 & 66 & 2,6 & 2 & 0,1 \\
\hline Tratamento cirúrgico periodontal (por sextante) & 7 & 0,3 & 3 & 0,1 & 131 & 5,1 & 30 & 1,6 \\
\hline Demais procedimentos periodontais & 0 & - & 5 & 0,2 & 7 & 0,3 & 1 & 0,1 \\
\hline Total & 2.770 & 100,0 & 2.640 & 100,0 & 2.573 & 100,0 & 1.861 & 100,0 \\
\hline \multicolumn{9}{|l|}{ Cirurgia oral menor } \\
\hline Remoção de dente retido (incluso/ impactado) & 89 & 15,9 & 379 & 17,4 & 246 & 23,9 & 106 & 12,5 \\
\hline Remoção de foco residual & 152 & 27,1 & 1.057 & 48,5 & 114 & 11,1 & 484 & 56,8 \\
\hline Exodontia de dente permante & 76 & 13,6 & 224 & 10,2 & 213 & 20,7 & 9 & 1,1 \\
\hline Excisão e sutura de lesões na boca & 139 & 24,8 & 1 & 0,1 & 186 & 18,1 & 18 & 2,1 \\
\hline Demais procedimentos cirúrgicos & 104 & 18,6 & 518 & 23,8 & 271 & 26,2 & 234 & 27,5 \\
\hline Total & 560 & 100,0 & 2.179 & 100,0 & 1.030 & 100,0 & 851 & 100,0 \\
\hline \multicolumn{9}{|l|}{ Endodontia } \\
\hline Obturação em dente permanente uni ou birradicular & 337 & 15,7 & 260 & 36,1 & 761 & 21,6 & 211 & 17,9 \\
\hline Obturação em dente permanente com três ou mais raízes & - & - & 232 & 32,1 & - & - & - & - \\
\hline Retratamento endodôntico & 279 & 12,9 & 91 & 12,6 & 66 & 1,8 & 33 & 2,8 \\
\hline $\begin{array}{l}\text { Acesso à polpa dentária e medicação e curativo de demora } \\
\text { (com ou sem preparo biomecânico) }\end{array}$ & 1.346 & 62,6 & 68 & 9,4 & 2.467 & 69,8 & 807 & 68,3 \\
\hline Demais procedimentos endodônticos & 190 & 8,8 & 71 & 9,8 & 236 & 6,8 & 130 & 11,0 \\
\hline Total & 2.152 & 100,0 & 722 & 100,0 & 3.530 & 100,0 & 1.181 & 100,0 \\
\hline
\end{tabular}

mas como não somos nós que agilizamos esses pacientes, eles chegam a nós através de consulta que é uma demanda que vem através da central de marcação (...) hoje, por exemplo, teve duas ausências..." (Endodontista, município A).

"Em média são oito pacientes, por sala, por turno. (...) mas os pacientes faltam muito. Depende da especialidade, às vezes, depende da especialidade e também do tempo" (Auxiliar de Saúde Bucal 1, município B).

"Fechou em cinco pacientes por turno, depois da reunião que na época teve (...) segundafeira mesmo, a gente atende cinco, mas a maioria não vem, aí a gente atende três, aí quando da terça feira, (...) os pacientes faltam muito, a gente tem que ficar ligando pro paciente para ele comparecer..."(Auxiliar de Saúde Bucal 2, município E).

Ainda assim, há a formulação de estratégias criativas para a garantia da boa utilização do serviço.

“Os pacientes são agendados por ordem de chegada. A gente confirma o atendimento das sete e meia às nove horas, ele deve estar presente.
Chega e confirma presença. Não são marcados por hora e ele tem que confirmar a consulta, ele pode sair, resolver outra coisa e retornar, não tem problema, mas a gente tem que ter controle dos pacientes que estão marcados porque, às vezes, quando falta o paciente a gente pode fazer uma abertura, uma instrumentação e concluir o fechamento do canal em uma só sessão" (Endodontista, município C).

Por outro lado, as diferentes tecnologias relacionadas à resolução do caso também têm interferido no número de marcações e tempo de consulta.

“...Três pacientes por turno, porque a gente procura dentro do possível instrumentar todo o canal e terminar na mesma sessão, quando o canal nos permite, pra que outras pessoas possam estar começando o tratamento, então a gente não coloca muitos pacientes, porque se não a gente vai ficar só fazendo acesso, instrumentando e não conclui, birradicular e unirradicular" (Endodontista, município B).

"Então minha cota é de 6 pacientes, 3 da central de marcação de consulta e 3 retornos. Eu gasto 
20, 30 minutos no máximo, 30 minutos no mais demorado" (Endodontista, município A).

"Quantos pacientes por turno? Cirurgia atende 3 pacientes cirúrgicos porque é 3o molar algo mais. Sim, então, por dia dependendo do procedimento de cada paciente. Especialidades? 12,10,8,9,10 pacientes. Só cirurgia que nós atendemos assim de 3 a 4 pessoas. Nós levamos 1 hora e alguma coisinha, dependendo do caso do paciente" (Auxiliar de Saúde Bucal 2, município B).

Alguns municípios adotam algum protocolo próprio para melhor articulação com a atenção primária, como definição de tipos de dente ou grau de lesão para referenciamento, com cotas por unidade. Outro aspecto a relatar se refere à subutilização do equipamento instalado, evidenciada nessa fala do coordenador.

“...Todos profissionais que lá trabalham [no CEO] tem consultório privado e você sabe 40 horas, em todos os lugares são no papel, o CEO nosso como é o tipo I, ele tem que ter 120 horas, com a soma de todos os profissionais. Então, nós ficamos de sete da manhã até uma da tarde. Então à tarde o CEO não funciona, eu acho que deveria funcionar, mas isso é uma outra questão, porque vai ter que contratar mais gente, tem que ter mais investimento, eu acho até que deveria funcionar à noite..." (Coordenador, município C).

"É [o horário de atendimento], isso varia mais da clínica, tem profissionais que começam a trabalhar mais tarde, eu mesmo começo a trabalhar a partir de 9 horas, pela manhã a partir de 9 horas, à tarde tem uns profissionais que combinam com os pacientes para chegar mais cedo" (Cirurgiãobuco-maxilo, município B).

Houve relatos de conflito e discordâncias sobre o melhor manejo de casos entre especialistas e profissionais da atenção primária. Por outro lado, os especialistas têm reconhecido as potencialidades da atenção primária.

"[O dentista da atenção primária] encaminha de forma errada, inadequadamente, sem adequação, sem orientação de higiene. Nesses casos a gente recebe o paciente, atende, só que a gente devolve, ele consegue adequar lá" (Endodontista, município C).

“Teoricamente, no protocolo que nós fizemos baseado em alguns protocolos, a parte de raspagem supra gengival seria feita nas unidades de saúde, mas alguns casos a gente faz aqui também" (Periodontista, município E).

"No consultório eu faço a anamnese do paciente e executo aquele procedimento, preencho a contra-referência, encaminho o paciente junto com a documentação dele. Aqui a gente não faz odontograma. O odontograma dele fica no posto, só vai ser executado o que foi solicitado na referência. A gente devolve pra o colega da unidade de atenção básica, complementar no odontograma, junto com as radiografias. Não fica, a parte de documentação, não fica nada aqui" (Cirurgiãobuco-maxilo, município D).

Houve relatos de resultados positivos na oferta do serviço especializado, a principal delas relacionada à redução da perda dental evitável.

“...A gente tem conseguido resultados bons, no sentido de estar salvando unidades dentárias que em outras ocasiões seriam perdidas, tanto na perio como na endodontia" (Periodontista, município A).

"Foram 336 de canais endodônticos, foi muito pouco, a gente pensou que fosse mais. Agora vamos olhar por outro lado, foram 336 dentes que deixamos de extrair, esse não extrair não tem preço. Então esse suporte que a gente dá na atenção básica ele tem que existir, tem que existir" (Coordenador, município A).

Entre os resultados, uma certa interface com o serviço odontológico privado é observada.

“Quando não tinha o CEO, o tratamento de periodontia era extração, estava com mobilidade, extraía, não tinha jeito, ele não podia ir para o consultório particular e na unidade básica não tinha como fazer, o profissional não tinha treinamento para fazer (...) Eu acho que, de certa forma, eu recebo paciente no CEO que já foi meu paciente no consultório" (Periodontista, município D).

“...Porque geralmente dente com endodontia precisa fazer um 'onlay', um 'bloquinho', uma 'coroa', então pelo menos diminui o custo para o paciente, diminui essa parte de endodontia que ele faz aqui, de adolescentes, a parte de trauma" (Endodontista, município C).

“Os pacientes não podiam pagar não é? Pagar pra fazer canal, aí não podia fazer cirurgia, tinha que se deslocar para fazer uma pequena cirurgia e faz aqui" (Auxiliar de Saúde Bucal, município C).

\section{Discussão}

Esse estudo revelou que, apesar de a oferta desse serviço especializado apresentar bons resultados na percepção dos profissionais executores dessa política - como a provável redução de perda dental e acesso a especialidades anteriormente restritas à Odontologia de Mercado -, há uma baixa taxa de utilização dos serviços públicos odontológicos especializados nos quatro CEOs analisados, conforme os padrões propostos pela Portaria GM no. 1.101/2002 20 e pela consulta aos especialistas, ou seja, a oferta disponível está, de fato, sendo subutilizada para um tipo de serviço essencial na garantia da integralidade da atenção à saúde bucal. 
Nesse sentido, a baixa taxa de utilização em algumas especialidades pode ser reveladora de problemas de gestão do serviço. Entre aqueles identificados nesse estudo, pode-se destacar a ausência de clareza sobre os padrões e metas propostos pelo serviço, inclusive no discurso dos próprios coordenadores, a constante falta de pacientes, que não são substituídos, além das diferentes tecnologias utilizadas por cada especialista, o que dificulta possíveis padronizações, a exemplo da endodontia, onde alguns especialistas optam por tratamentos longos e completos por sessão (abertura e fechamento), enquanto outros elegem o modelo de tratamentos curtos, mas fracionados.

Na Bahia, no período entre 2008 e 2009, a oferta de atenção especializada se ampliou consideravelmente com a habilitação de 71 CEOs. Contudo, a análise da produção ambulatorial desse tipo de serviço, com proxy do conceito de utilização aponta para um aumento dos procedimentos de cirurgia oral menor $(30,6 \%)$, mas redução dos procedimentos de endodontia $(-21 \%)$ e periodontais $(-45,9 \%)$ (Departamento de Informática do SUS. Sistema de Informação Ambulatorial do SUS. http://www.datasus.gov. br, acessado em 02/Fev/2010).

Há conflitos importantes na integração entre a atenção básica e atenção especializada, não apenas na indicação adequada de casos para esse nível de atenção, bem como na chegada do paciente ao CEO sem a devida adequação e promoção de saúde bucal, funções da atenção primária. Os autores consideram esta uma das questões mais críticas na coordenação da atenção, ou seja, a interface da atenção primária com a atenção secundária ou terciária 10,25.

Estudo na Noruega aponta que um sistema de incentivos baseado na remuneração por captação levou a um aumento do número de indivíduos sob vigilância na atenção primária, sem reduzir a qualidade ${ }^{3}$. No caso da atenção secundária, um sistema de remuneração fixa pode estar desencorajando os profissionais a cumprirem metas mínimas de oferta de serviços.

As baixas e diferenciadas taxas de utilização reveladas entre os serviços de atenção especializada analisados neste estudo podem sugerir também o não cumprimento da carga horária real dos profissionais. Cabe destacar inclusive que os profissionais lotados na atenção pública especializada têm dupla militância, também revelada por esta investigação e, embora a atuação profissional no serviço público e o exercício privado da odontologia não sejam conflitantes, é um desafio para os gestores públicos na medida em que a lógica dominante do campo privado odontológico está presente nas aspirações dos profissionais, que não se consideram muitas vezes ajustados ou incorporados ao campo da saúde pública 6,26.

Quanto à acessibilidade ao serviço, a reorganização do fluxo de pacientes e dos processos de trabalho nos serviços de atenção secundária poderia incluir redução de barreiras organizacionais como a implantação do agendamento prévio, bem como uma lista de espera diária para substituição de faltosos ou com marcação extra, considerando a provável falta de pacientes 27,28 , o que é amplamente recomendado para aproveitamento da oferta disponível e redução de consultas de retorno, que muitas vezes são barreiras importantes porque muitos retornos podem significar a não procura posterior do serviço pelo paciente por falta de dinheiro para o transporte. Ferreira \& Loureiro 12 sugerem a redução da barreira de acesso com o aumento do tempo de consulta, implicando menor número de visitas, de modo a reduzir o custo para o paciente.

Nos CEOs analisados, não houve adequação da oferta às necessidades epidemiológicas, especialmente na endodontia, haja vista que a maioria dos CEOs ofertou apenas endodontia de uni e bi-radiculares (Tabela 4). Diversos estudos epidemiológicos 29,30 apontam que são os molares as unidades mais acometidas por perda dental decorrente da cárie dentária e, nesse sentido, deveriam ser priorizados, diferentemente do que foi revelado no presente estudo.

Outra discussão relevante a ser enfrentada pela PNSB se refere aos limites entre a atenção primária e secundária em processo de superação no sistema de saúde inglês (National Health System), onde cada vez mais muitos procedimentos ditos secundários têm sido prestados por dentistas com interesse ou habilidade especial naquela área na própria atenção primária 6,31.

\section{Considerações finais}

As altas taxas de utilização identificadas com o padrão proposto pelas Portarias GM $n^{\circ}$. $1.571 / 200421$ e GM $n^{o}$. 1.572/2004 22 apontam uma inadequação da formulação desse padrão, baseado na série histórica de produção, seguindo o proposto pela Portaria MPAS $n^{\circ}$. 3.046/1982 32 . Nesse sentido, a avaliação entre a oferta e utilização dos serviços odontológicos aponta para a necessidade de revisão das portarias do CEO especialmente porque nenhuma relação entre tipo de CEO, número de equipamentos odontológicos instalados e a oferta potencial proposta foi identificada. Neste sentido, sugere-se um padrão de oferta baseado no número de profissionais/ consultórios e conforme consulta a especialistas realizada no presente estudo, que parecem ser 
mais adequadas ao processo de trabalho em ambulatório de especialidades. Estudo de Antunes \& Narvai 33 já aponta para uma subutilização de consultórios odontológicos públicos instalados, também revelada no discurso dos entrevistados e observação pela presente investigação.

A presente investigação foi exploratória e identificou como possíveis fatores relacionados às baixas taxas de utilização as barreiras de acesso do próprio serviço de saúde, como a falta de um sistema de lista de espera automática para substituição de faltosos 27,28 e sugere que a implantação dos CEOs deve estar articulada com um sistema de gestão com a definição clara das metas de oferta de procedimentos por especialidade. A construção de protocolos de referência bem definidos podem, também, contribuir para uma melhor utilização dos serviços especializados reduzindo a possibilidade de realização de procedimentos básicos nos CEOs, o que pode justificar a taxa de utilização satisfatória de periodontia nos municípios estudados em quase todos os critérios adotados para a análise.

Por fim, cabe destacar que pelo caráter exploratório deste estudo não foi possível o aprofundamento na análise por tipo de especialidade e o mesmo apresenta limitações por tratar da avaliação de apenas quatro CEOs do Estado da Bahia. Sugere-se a realização de estudos capazes de preencher as lacunas ainda existentes. Estudos que possam incorporar a discussão do custobenefício no uso de diferentes tipos de tecnologias como no caso da endodontia e periodontia são indicados, bem como diferentes formas de organização do serviço que possibilitem maior utilização da oferta disponível, quer sejam estas alternativas relacionadas a diferenças de remuneração, articulação com atenção primária e adequação da oferta especializada às necessidades de serviços de saúde da população.

\section{Resumo}

O estudo visou avaliar a taxa de utilização dos serviços odontológicos especializados de quatro CEOs da Bahia, Brasil, identificando fatores relacionados. Trata-se de uma pesquisa avaliativa onde a etapa quantitativa foi conduzida através de um estudo transversal utilizando dados secundários do Sistema de Informações Ambulatoriais do Sistema Único de Saúde (SIASUS) e a qualitativa foi a partir de vinte entrevistas semi-estruturadas junto aos coordenadores e executores, além de observação in loco do serviço. O estudo revelou bons resultados na oferta desse serviço na percepção dos profissionais, mas há uma baixa taxa de utilização. A baixa taxa de utilização revela barreiras de acesso do próprio serviço de saúde, como a ausência de padrões e metas de desempenho por especialidade, a falta dos pacientes, que não são substituídos, além das diferentes tecnologias utilizadas por cada especialista. Sugerem-se estudos que analisem diferentes formas de organização do serviço que tenham possibilitado maior utilização da oferta disponível, como diferenças de remuneração, articulação com atenção primária e adequação da oferta às necessidades de saúde populacionais.

Especialidades Odontológicas; Serviços de Saúde Bucal; Avaliação em Saúde 


\section{Colaboradores}

S. C. L. Chaves colaborou na formulação da pergunta desenho metodológico e coleta de dados, analisou os dados e elaborou a versão final do artigo. D. N. Cruz colaborou na formulação da pergunta de investigação, realizou coleta e análise de dados e contribuiu na elaboração do artigo e na sua revisão. S. G. Barros contribuiu na formulação da consulta aos experts, realizou coleta de dados e revisou o texto final do artigo. A. L. Figueiredo participou na formulação do desenho do estudo, colaborou na coleta e análise dos dados e contribuiu com a versão final do artigo.

\section{Agradecimentos}

Agradecemos a Thales Souza Nunes e Bárbara Laisa Alves Moura pela colaboração na coleta de dados. A Fundação de Amparo à Pesquisa do Estado da Bahia (FAPESB, edital PP/SUS 2007).

\section{Referências}

1. Beazoglou T, Heffley D, Brown LJ, Bailit H. The importance of productivity in estimating need for dentists. J Am Dent Assoc 2002; 133:1399-404.

2. Grytten J. Models for financing dental services. A review. Community Dent Health 2005; 22:75-85.

3. Grytten J, Holst D, Skau I. Incentives and remuneration systems in dental services. Int J Health Care Finance Econ 2009; 9:259-78.

4. Ribeiro-Sobrinho C, Souza LEPF, Chaves SCL. Avaliação da cobertura do Serviço Odontológico da Polícia Militar em Salvador, Bahia, Brasil. Cad Saúde Pública 2008; 24:295-302.

5. Guay AH. Access to dental care: the triad of essential factors in access-to-care programs. J Am Dent Assoc 2004; 135:779-85.

6. Chaves SCL, Vieira-da-Silva LM. Atenção à saúde bucal e a descentralização da saúde no Brasil: estudo de dois casos exemplares no Estado da Bahia. Cad Saúde Pública 2007; 23:1119-31.

7. Matos DL, Lima-Costa MFF, Guerra HL, Marcenes W. Projeto Bambuí: estudo de base populacional dos fatores associados com o uso regular de serviços odontológicos em adultos. Cad Saúde Pública 2001; 17:661-8.
8. Grytten J, Holst D, Grytten L. Supply decisions among dentists working within a fixed-fee system of dental care provision. J Public Health Dent 1992; 52:204-9.

9. Mendoza-Sassi R, Béria JU. Utilización de los servicios de salud: una revisión sistemática sobre los factores relacionados. Cad Saúde Pública 2001; 17:819-32.

10. Morris AJ, Burke FJT. Primary and secondary dental care: how ideal is the interface? Br Dent J 2001; 191:666-70.

11. Figueiredo N, Goes PSA. Construção da atenção secundária em saúde bucal: um estudo sobre os Centros de Especialidades Odontológicas em Pernambuco, Brasil. Cad Saúde Pública 2009; 25: 259-67.

12. Ferreira CA, Loureiro CA. Custos para implantação e operação de serviço de saúde bucal na perspectiva do serviço e da sociedade. Cad Saúde Pública 2008; 24:2071-80.

13. Pucca Junior GA, Costa JF, Chagas LD, Silvestre RM. Oral health policies in Brazil. Braz Oral Res 2009; 23 Suppl 1:9-16. 
14. World Health Organization. Oral health for the 21st century. Geneva: World Health Organization; 1994.

15. Barros AJD, Bertoldi AD. Desigualdades na utilização e no acesso a serviços odontológicos: uma avaliação em nível nacional. Ciênc Saúde Coletiva 2002; 7:709-17.

16. Watt RG, Sheiham A. Inequalities in oral health: a review of the evidence and recommendations for action. Br Dent J 1999; 187:6-12.

17. Evans RG, Morris L, Marmor T. Producir salud, consumir asistencia sanitaria. In: Evans RG, organizador. ¿Porqué alguna gente está sana y otra no? Madrid: Editorial Díaz de Santos; 1996. p. 29-70.

18. Bauer MW, Gaskell G. Pesquisa qualitativa com texto, imagem e som. Petrópolis: Editora Vozes; 2002.

19. Vieira-da-Silva LM. Conceitos, abordagens e estratégias para a avaliação em saúde. In: Hartz ZMA, Vieira-da-Silva LM, organizadoras. Avaliação em saúde: dos modelos teóricos à prática na avaliação de programas e sistemas de saúde. Salvador: Editora EDUFBA/Rio de Janeiro: Editora Fiocruz; 2005. p. 15-39.

20. Ministério da Saúde. Portaria nº ${ }^{\circ} 1.101$, de 12 de junho de 2002. Diário Oficial da União 2002; 13 jun.

21. Ministério da Saúde. Portaria no ${ }^{\circ} .571$, de 29 de julho de 2004. Estabelece o pagamento de próteses dentárias totais em Laboratórios Regionais de Próteses Dentárias - LRPD. Diário Oficial da União 2004; 30 jul.

22. Ministério da Saúde. Portaria $\mathrm{n}^{\circ}$. 1.572, de 29 de julho de 2004. Estabelece o financiamento dos Centros de Especialidades Odontológicas - CEO. Diário Oficial da União 2004; 30 jul.

23. Donabedian A. Methods of deriving the criteria. In: Donabedian A, editor. Explorations in quality assessment and monitoring. Ann Arbor: Health Administration Press; 1982. p. 504-70.

24. Jones J, Hunter D. Using the Delphi and nominal group technique in health services research. In: Pope C, Mays N, editors. Qualitative research in health care. London: BMJ Books; 2000. p. 40-9.
25. Starfield B. Coordenação da atenção: juntando tudo. In: Starfield B, organizador. Atenção primária: equilíbrio entre necessidades de saúde, serviços e tecnologia. Brasília: UNESCO Brasil/Ministério da Saúde; 2004. p. 365-415.

26. Chaves MC, Miranda AS. Discursos de cirurgiõesdentistas do Programa Saúde da Família: crise e mudança de habitus na saúde pública. Interface Comun Saúde Educ 2008; 12:153-67.

27. Vieira-da-Silva LM, Chaves SC, Esperidião MA, Lopes-Martinho RM. Accessibility to primary healthcare in the capital city of a northeastern state of Brazil: an evaluation of the results of a programme. J Epidemiol Community Health 2010; 64:1100-5.

28. Mehrotra A, Keehl-Markowitz, L, Ayanian, J. Implementing open-access scheduling of visits in primary care practices: a cautionary tale. Ann Intern Med 2008; 148:915-22.

29. Barbato PR Nagano HCM, Zanchet FN, Boing AF, Peres MA. Perdas dentárias e fatores sociais, demográficos e de serviços associados em adul tos brasileiros: uma análise dos dados do Estudo Epidemiológico Nacional (Projeto SB Brasil 20022003). Cad Saúde Pública 2007; 23:1803-14.

30. Narvai PC, Frazão P, Roncalli AG, Antunes JLF. Cárie dentária no Brasil: declínio, polarização, iniqüidade e exclusão social. Rev Panam Salud Pública 2006; 19:385-93.

31. Morris S, Sutton M, Gravelle H. Inequity and inequality in the use of health care in England: an empirical investigation. Soc Sci Med 2005; 60:125166.

32. Ministério da Previdência e Assistência Social. Portaria nº. 3046. Diário Oficial da União 1982; 20 jul.

33. Antunes JLF, Narvai PC. Políticas de saúde bucal no Brasil e seu impacto sobre as desigualdades em saúde. Rev Saúde Pública 2010; 44:360-5.

Recebido em 29/Mai/2010

Versão final reapresentada em 04/Out/2010 Aprovado em 06/Out/2010 